\title{
A Note on the Power Spectrum of the Iterates of Feigenbaum's Function*
}

\author{
P. Collet ${ }^{1}$, J.-P. Eckmann ${ }^{2}$ and L. Thomas ${ }^{2}$ \\ 1 Ecole Polytechnique, Paris, France \\ 2 Département de Physique Théorique, Université de Genève, CH-1211 Genève 4, Switzerland
}

\begin{abstract}
We give rigorous bounds on the scaling behaviour of the power spectrum for Feigenbaum's map.
\end{abstract}

Recently, estimates have been given concerning the scaling behaviour of the power spectrum for the Feigenbaum map, Feigenbaum [1,2], Nauenberg and Rudnick [3]. Since these estimates are based on somewhat heuristic arguments, it might be useful to give rigorous bounds on this scaling behavior. The purpose of this note is to give such bounds.

We denote by $\varphi$ the Feigenbaum function, defined [4] by the relations

$$
\varphi(\varphi(\lambda x))=-\lambda \varphi(x), \quad \lambda=-\varphi(1), \quad \varphi(0)=1,
$$

$\varphi$ analytic. For every diadic rational $q=k / 2^{N}, k$ odd, we define the amplitudes

$$
a(q)=\lim _{p \rightarrow \infty} \frac{1}{p} \sum_{j=0}^{p-1} \exp \left(2 \pi i k j / 2^{N}\right) \varphi^{j}(0) .^{1}
$$

(The limit exists since $\left\{\varphi^{j}(0)\right\}$ is almost periodic.) We define the averaged square amplitude $A_{N}$ at level $N$ as

$$
A_{N}=\frac{1}{2^{N-1}} \sum_{k=0}^{2^{N-1}-1}\left|a\left((2 k+1) / 2^{N}\right)\right|^{2} .
$$

Theorem. For all $M>0$, one has the bound

$$
\left(\frac{\lambda^{2}}{4}\right)^{M} \leqq \frac{A_{N+M}}{A_{N}}<\left(\frac{\lambda^{2}}{4}\right)^{M} X Y^{M},
$$

* Work partially supported by the Fonds National Suisse

$1 \quad \varphi^{j}(x)=\varphi\left(\varphi^{j-1}(x)\right), \quad j>0, \quad \varphi^{0}(x)=x$ 
with $X<14.9, Y<1.109$. When $M=1$, the upper bound can be replaced by $2.52 \cdot\left(\lambda^{2} / 4\right)$.

We give analytic expressions for $X, Y$ in the proof.

Remarks. Successive averaged square amplitudes decrease asymptotically by between 13.45 and 14 decibels (i.e. a factor $F, 22.3<F<25.1$ ). This result should be compared with that of Feigenbaum $[1,2]$ who considers a family $\left\{\varphi_{N}\right\}$ of maps, $\varphi_{N}$ having a stable period of period $2^{N}, \lim _{N \rightarrow \infty} \varphi_{N}=\varphi$, and satisfying $\varphi_{N}\left(\varphi_{N}(\lambda x)\right)=-\lambda \varphi_{N-1}(x)$. By assuming the existence of a scaling function relating $\varphi_{N}^{j}(0)-\varphi_{N}^{j+2^{N-1}}(0)$ to $\varphi_{N+1}^{j}(0)-\varphi_{N+1}^{j+2^{N}}(0)$, he obtains a scaling relation between successive Fourier amplitudes $a_{N}(\cdot), a_{N+1}(\cdot)$ which leads to a decrease of about $16.3 \mathrm{db}$ for successive averaged square amplitudes. (We emphasize that our result is a statement about the function $\varphi$ only.) Nauenberg and Rudnick [3] have investigated a sequence analogous to $A_{N}$ defined above. They find a decrease of about $13.2 \mathrm{db}$ both by numerical means, and by exploiting a self-similarity property in the shape of $\varphi$ and its iterates.

Proof. Using the definitions (2), (3), it is easy to see that

$$
A_{N}=\lim _{p \rightarrow \infty} \frac{1}{4^{N} p^{2}} \sum_{j=0}^{2^{N-1}-1}\left(\sum_{s=0}^{p-1} \varphi^{j+s 2^{N}}(0)-\varphi^{j+s 2^{N}+2^{N-1}}(0)\right)^{2} .
$$

If we neglect all terms in (4) with odd $j$, and use the identity (1) then we see that

$$
A_{N} \geqq \frac{\lambda^{2}}{4} A_{N-1} \text {, }
$$

which is the lower bound in the theorem.

The upper bound is more delicate. In order to analyze the squares in (4), we first assert that, for fixed $j<2^{N-1}$, the sign of $\varphi^{j+s 2^{N}}(0)-\varphi^{j+s 2^{N}+2^{N-1}}(0)$ is independent of $s$. To prove this, we observe first that $\varphi^{j}$ has no zero on $\left(0, \lambda^{N-1}\right)$ for $j=1, \ldots, 2^{N-1}-1$. For, if $0<x<\lambda^{N-1}$ and if $\varphi^{j}(x)=0$, we would find that $\varphi^{2^{N-1}}\left(\lambda^{N-1} t\right)=(-\lambda)^{N-1} \varphi(t)$ would have a zero derivative at $t \in(0,1)$, which contradicts $\varphi^{\prime}(t) \neq 0$ if $t \neq 0$. Using the chain rule of differentiation, we see that $\varphi^{j}$ is monotone on $\left[0, \lambda^{N-1}\right]$. The assertion follows now from the identities

$$
\varphi^{j+s 2^{N}}(0)-\varphi^{j+s 2^{N}+2^{N-1}}(0)=\varphi^{j}\left(\lambda^{N-1} \varphi^{2 s}(0)\right)-\varphi^{j}\left(\lambda^{N-1} \varphi^{2 s}(1)\right),
$$

and $\varphi^{2 s}(1)>\left|\varphi^{2 s}(0)\right|$ for all $s \geqq 0$, a general property of the solution to Eq. (1).

We fix now $K$ and $M, K>M$ and compare $A_{K}$ to $A_{M}$. We use the notation $\sum_{j}^{L}$ for $\sum_{j=0}^{2^{L}-1}$. We have, from (4),

$$
\begin{gathered}
A_{K}=\lim _{p \rightarrow \infty} \frac{1}{4^{K} p^{2}} \sum_{k}^{M} \sum_{\ell}^{K-M-1}\left(\sum_{s=0}^{p-1} \varphi^{k+\ell 2^{M}+s 2^{K}}(0)-\varphi^{k+\ell 2^{M}+\left(s+\frac{1}{2}\right) 2^{K}}(0)\right)^{2} \\
=\lim _{p \rightarrow \infty} \frac{1}{4^{K} p^{2}} \sum_{k}^{M} \sum_{\ell}^{K-M-1}\left(\sum_{s=0}^{p-1}\left(\frac{d}{d x} \varphi^{k}\right)\left(z_{k, \ell, s}^{K, M}\right)\right. \\
\left.\cdot\left[\varphi^{\ell 2^{M}+s 2^{K}}(0)-\varphi^{\ell 2^{M}+\left(s+\frac{1}{2}\right) 2^{K}}(0)\right]\right)^{2}
\end{gathered}
$$


where

$$
\begin{aligned}
z_{k, \ell, s}^{K, M} \in\left[\varphi^{\ell 2^{M}+s 2^{K}}(0) ; \varphi^{\ell 2^{M}+\left(s+\frac{1}{2}\right) 2^{K}}(0)\right] \\
=\left[\lambda^{M} \varphi^{\ell+s 2^{K-M}}(0) ; \lambda^{M} \varphi^{\ell+\left(s+\frac{1}{2}\right) 2^{K-M}}(0)\right],
\end{aligned}
$$

and $[a ; b]$ denotes the interval with endpoints $a, b$ (any order). Using the constancy of sign discussed above, and (1), we can bound (5) by

where

$$
A_{K} \leqq\left(\frac{\lambda^{2}}{4}\right)^{M} D_{M} A_{K-M}
$$

$$
\begin{aligned}
D_{M} & =\sum_{k}^{M} \sup _{\ell, s}\left|\left(\frac{d}{d x} \varphi^{k}\right)\left(z_{k, \ell, s}^{K, M}\right)\right|^{2} \\
& \leqq \sum_{k}^{M} \sup _{t \in[-1,1]}\left|\left(\frac{d}{d x} \varphi^{k}\right)\left(\lambda^{M} t\right)\right|^{2}=: \Delta_{M}^{\prime} .
\end{aligned}
$$

We have used (6) in the last inequality.

We shall now estimate $\Delta_{M}^{\prime}$. Using repeatedly Eq. (1) and the binary decomposition of $k, 1 \leqq k<2^{M}$,

$$
k=\sum_{j=0}^{r-1} 2 \sum_{q=0}^{J} \sigma_{q}, \quad r=r(k)
$$

$\sigma_{q} \geqq 1$ if $q \geqq 1, \sigma_{0} \geqq 0$, we find

$$
\begin{aligned}
& \left|\left(\frac{d}{d x} \varphi^{k}\right)\left(\lambda^{M} t\right)\right|=\lambda^{-M}\left|\frac{d}{d t} \varphi^{k}\left(\lambda^{M} t\right)\right| \\
& =\lambda^{-M}\left|\frac{d}{d t} \lambda^{\sigma_{0}} \varphi\left(\lambda^{\sigma_{1}} \ldots \varphi\left(\lambda^{\sigma_{r}} t\right) \ldots\right)\right|,
\end{aligned}
$$

where $\sigma_{0}+\ldots+\sigma_{r}=M$, with $\sigma_{r} \geqq 1$. By the chain rule of differentiation, we get

$$
\left|\left(\frac{d}{d x} \varphi^{k}\right)\left(\lambda^{M} t\right)\right|=\left|\varphi^{\prime}\left(\lambda^{\sigma_{r}} t\right)\right| \prod_{j=1}^{r-1}\left|\varphi^{\prime}\left(\lambda^{\sigma_{J}} \varphi\left(\lambda^{\sigma_{j+1}} v_{j}\right)\right)\right|
$$

with $\left|v_{j}\right| \leqq 1$. From the known coefficients of $\varphi$, see [5], [4] we conclude that $\operatorname{sign} \varphi^{\prime \prime \prime}(x)=\operatorname{sign} x$ if $|x| \leqq \lambda$. This, and the concavity of $\varphi$ imply $-\varphi^{\prime \prime}(0)$ $=\sup _{|x|<\lambda}\left|\varphi^{\prime \prime}(x)\right|$. It follows that for $|v| \leqq 1$,

$$
\left|\varphi^{\prime}\left(\lambda^{\sigma} \varphi\left(\lambda^{\tau} \nu\right)\right)\right| \leqq \gamma \lambda^{\sigma}\left|\varphi\left(\lambda^{\tau}\right)\right|
$$

where $\gamma=\left|\varphi^{\prime \prime}(0)\right|<3.056$. It is now convenient to define $b_{\sigma}$ as follows

$$
b_{\sigma}=\left\{\begin{array}{lll}
\gamma^{2} & \text { if } & \sigma>1 \\
\gamma^{2} \varphi(\lambda)^{2} & \text { if } & \sigma=1
\end{array}\right.
$$

Note that $0.7589<|\varphi(\lambda)|<0.759$. Then we obtain

$$
\left|\left(\frac{d}{d x} \varphi^{k}\right)\left(\lambda^{M} t\right)\right|^{2} \leqq \lambda^{2\left(M-\sigma_{0}\right)} \varphi(\lambda)^{-2} \prod_{j=1}^{r} b_{\sigma_{J}},
$$


and hence

$$
\begin{aligned}
D_{M} & \leqq \Delta_{M}^{\prime} \\
& \leqq 1+\varphi(\lambda)^{-2} \sum_{\substack{r=1 \\
r=1}}^{M} \sum_{\substack{\sigma_{0}+\ldots+\sigma_{r}=M \\
\sigma_{i} \geqq 1 \text { if } i \geqq 1 \\
\sigma_{0} \geqq 0}} \lambda^{2\left(M-\sigma_{0}\right)} \prod_{j=1}^{r} b_{\sigma_{J}} \\
& =1+\varphi(\lambda)^{-2} \sum_{\sigma_{0}=0}^{M-1} \lambda^{2\left(M-\sigma_{0}\right)} \sum_{r=1}^{M-\sigma_{0}} \sum_{\sigma_{1}+\ldots+\sigma_{r}=M-\sigma_{0}} \prod_{j=1}^{r} b_{\sigma_{j}}=: \Delta_{M} .
\end{aligned}
$$

In order to estimate $\Delta_{M}$ we introduce

$$
C_{L}=\sum_{r=1}^{L} \sum_{\substack{\sigma_{1}+\ldots+\sigma_{r}=L \\ \sigma_{l} \geqq 1 \forall i}} \prod_{j=1}^{r} b_{\sigma_{J}}, \quad L>0,
$$

$C_{0}=1, C_{L}=0$ if $L<O$. Then we have

$$
C_{L+1}=\gamma^{2} \varphi(\lambda)^{2} C_{L}+\gamma^{2} \sum_{j=1}^{\infty} C_{L-j}
$$

It follows that the generating function $F(x)=\sum_{j=0}^{\infty} C_{j} x^{j}$ equals

$$
F(x)=\frac{1-x}{1-x\left(1+\gamma^{2} \varphi(\lambda)^{2}\right)-x^{2} \gamma^{2}\left(1-\varphi(\lambda)^{2}\right)} .
$$

Thus

$$
F(x)=\frac{\alpha_{+}}{x-x_{+}}+\frac{\alpha_{-}}{x-x_{-}},
$$

where $x_{ \pm}$are the roots of the denominator of (10), $x_{+}>x_{-}$and

$$
\alpha_{ \pm}=\frac{x_{ \pm}-1}{x_{ \pm}-x_{\mp}} \cdot \frac{1}{\gamma^{2}(1-\varphi(\lambda))} \text {. }
$$

We find from (11),

$$
C_{L}=-\frac{\alpha_{+}}{x_{+}^{L+1}}-\frac{\alpha_{-}}{x_{-}^{L+1}}
$$

Substituting back into (9), we see that

$$
\begin{aligned}
D_{M} & <\Delta_{M} \\
& =1+\varphi(\lambda)^{-2} \sum_{\sigma_{0}=0}^{M-1} \lambda^{2\left(M-\sigma_{0}\right)} C_{M-\sigma_{0}} \\
& =1-\frac{\lambda^{2}}{\varphi(\lambda)^{2}}\left\{\frac{\alpha_{+}}{x_{+}^{2}\left(\frac{\lambda^{2}}{x_{+}}-1\right)}\left(\left(\frac{\lambda^{2}}{x_{+}}\right)^{M}-1\right)+\frac{\alpha_{-}}{x_{-}^{2}\left(\frac{\lambda^{2}}{x_{-}}-1\right)}\left(\left(\frac{\lambda^{2}}{x_{-}}\right)^{M}-1\right)\right\} .
\end{aligned}
$$


Substituting the bounds for $\lambda, \varphi(\lambda)$ and $\varphi^{\prime \prime}(0)$, we see that

$$
\begin{aligned}
D_{M} & <1-\frac{\lambda^{2}}{\varphi(\lambda)^{2}} \frac{\alpha_{+}}{x_{+}^{2}\left(\frac{\lambda^{2}}{x_{+}}-1\right)}\left(\frac{\lambda^{2}}{x_{+}}\right)^{M} \\
& <1+13.996 \cdot(1.10883)^{M} .
\end{aligned}
$$

This combined with Eq. (7) proves the theorem. For small $M$, one can get better bounds by using (13) directly; e.g. $D_{1}<2.493$. A slight further improvement can be obtained by replacing the right hand side of (8) by $\lambda^{2\left(M-\sigma_{0}\right)} \gamma^{2} \prod_{j=2}^{r} b_{\sigma_{j}}$, yielding 12.31 instead of 13.996 in Eq. (14).

\section{References}

1. Feigenbaum, M.J.: Phys. Lett. 74A, 375-378 (1979)

2. Feigenbaum, M.J.: Commun. Math. Phys. 77, 65-86 (1980)

3. Nauenberg, M., Rudnick, J.: Universality and the power spectrum at the onset of chaos. Preprint UCSC (1981)

4. Campanino, M., Epstein, H., Ruelle, D.: On Feigenbaum's functional equation $\varphi(\varphi(\lambda x))=$ $-\lambda \varphi(x)$. Preprint IHES (1980),

Campanino, M., Epstein, H.: On the existence of Feigenbaum's fixed point. Commun. Math. Phys. 79, 261-302 (1981)

Lanford, O.E.: A computer assisted proof of the Feigenbaum conjectures Preprint IHES 1981

5. Feigenbaum, M.: Qualitative universality for a class of nonlinear transformations. J. Stat. Phys. 19, 25-52 (1978); 21, 669-706 (1979)

Communicated by D. Ruelle

Received April 22, 1981 
